
\title{
R Research Square \\ Clinical Characteristics, Radiological Features, And Disease Severity Of Bronchiectasis According To The Spirometric Pattern
}

\section{Sun-Hyung Kim}

Chungbuk National University Hospital

\section{Bumhee Yang}

Chungbuk National University Hospital

Jin Young Yoo

Chungbuk National University Hospital

Jun Yeun Cho

Chungbuk National University Hospital

Hyeran Kang

Chungbuk National University Hospital

Yoon Mi Shin

Chungbuk National University Hospital

\section{Eung-Gook Kim}

Chungbuk National University College of Medicine

Ki Man Lee

Chungbuk National University Hospital

Kang Hyeon Choe ( $\nabla$ choekh@chungbuk.ac.kr)

Chungbuk National University Hospital

\section{Research Article}

Keywords:

Posted Date: February 4th, 2022

DOI: https://doi.org/10.21203/rs.3.rs-1256035/v1

License: (c) (1) This work is licensed under a Creative Commons Attribution 4.0 International License. Read Full License 


\section{Abstract}

Bronchiectasis show various ventilatory disorders in pulmonary function. The characteristics and severity of patients with bronchiectasis according to these pulmonary dysfunctions are still very limited. This study aimed to evaluate the clinical, radiologic feature and the disease severity of patients with bronchiectasis according to spirometric patterns. We retrospectively evaluated 506 patients with bronchiectasis who underwent pulmonary lung function test (PFT) at a referral hospital between 2014 to 2021. Cylindrical type was the most common (70.8\%) type of bronchiectasis on chest CT, and $70 \%$ of patients had bilateral lung involvement. Of the 506 patients, Obstructive ventilatory disorder was the most common (51.6\%), followed by normal ventilation (30\%) and restrictive ventilatory disorder (18.4\%). mMRC was highest in patients with obstructive ventilatory disorders, Modified Reiff score (6 (3-10), $P<$ $0.001)$, FACED score (3 (1-4), $P<0.001)$, and BSI score (8 (5-11), $P<0.001)$ also showed significantly highest values of obstructive ventilatory disorder rather than restrictive ventilatory disorder and normal ventilation. More than half of patients with bronchiectasis had obstructive ventilatory disorder. Bronchiectasis with obstructive ventilatory disorders has more dyspnea symptom, more disease severity and more radiologic severity. There was no significant association between spirometric pattern and radiologic type, but the more severe the radiologic severity, the more severe the lung function impairment.

\section{Introduction}

Non-cystic fibrosis bronchiectasis (hereafter referred to as bronchiectasis) is a chronic respiratory disease characterized by permanent dilatation of the bronchi and chronic respiratory symptoms, such as cough, expectoration of sputum, and dyspnea. The inherent dysfunction in mucociliary clearance leads to persistent bacterial infection, chronic inflammation of the bronchial tree, and progressive tissue destruction. Thus, persistent airway destruction leads to decreased lung function and respiratory failure ${ }^{1}$ 2 .

Previous studies have reported that the morphological changes in bronchiectasis are associated with pulmonary dysfunction ${ }^{3-5}$. Research suggests that the development of bronchiectasis is promoted by the early involvement of the lymphoid follicles in the small airways, which gradually leads to the obstruction of the more distal airways ${ }^{6}$. Accordingly, it has been known that bronchiectasis is accompanied by airflow limitation 7 . As a result, forced expiratory volume in 1 second (FEV1) was used to evaluate pulmonary dysfunction in patients with bronchiectasis, as a same line, $\mathrm{FEV}_{1}$ was included in Bronchiectasis Severity Index (BSI) score and FACED (FEV 1 , Age, Chronic colonization, Extension, and Dyspnea) score to evaluate the severity and prognosis of patient with bronchiectasis ${ }^{8,9}$. Moreover, recent studies have reported that patients with bronchiectasis exhibit various ventilatory disorders on pulmonary function tests, such as obstructive, restrictive, and mixed ventilatory disorder ${ }^{4,10-12}$. Although various ventilatory disorders of bronchiectasis have been reported as described above, studies on the characteristics and severity of patients with bronchiectasis according to these pulmonary dysfunctions are still very limited. 
Hence, we investigated the clinical and radiological features of patients with bronchiectasis according to the spirometric pattern. Moreover, we further evaluated the disease severity in patients with bronchiectasis according to spirometric patterns.

\section{Results}

\section{Baseline characteristics}

The baseline characteristics of 506 patients with bronchiectasis are presented in Table 1. The median age was 66 years (IQR, 53-79 years), and 254 (50.2\%) patients were male. The median body mass index (BMI) was 23.0 (IQR, 19.3-26.7). Current and ex-smokers accounted for $34.8 \%$ of the study population, and $23.7 \%$ of patients had a history of tuberculosis. The distribution of comorbidities in patients with bronchiectasis was as follows: chronic obstructive pulmonary disease (COPD) (51.6\%), followed by cardiovascular disease (37.7\%), asthma (16.2\%), diabetes mellitus (12.6\%), and malignancy $(12.6 \%)$. Cylindrical bronchiectasis was the most common (70.8\%) morphology on chest $\mathrm{CT}$, and $70 \%$ of patients exhibited bilateral lung involvement. The pulmonary function parameters were as follows: FVC \% predicted, 84\% (70-95); $\mathrm{FEV}_{1} / \mathrm{FVC}, 73 \%$ (56-90); and FEV1\% predicted, 69\% (55-78). Microorganisms were identified in $32.4 \%$ of patients: Pseudomonas aeruginosa (18.0\%) was the most frequently identified species, followed by Klebsiella pneumoniae (8.5\%), and Staphylococcus aureus (4.9\%). NTM were identified in $10.1 \%$ of patients, of which Mycobacterium avium complex (MAC) was the most common type (8.3\%). 
Table 1

Baseline characteristics of patients with bronchiectasis

\section{Variables}

Age, years

Sex, male

BMI $\left(\mathrm{kg} / \mathrm{m}^{2}\right)$

Smoking history

Current or ex-smoker

Previous history of TB

Previous history of pertussis

Comorbidities

COPD

Asthma

Cardiovascular disease

Diabetes mellitus

Chronic liver disease

Chronic kidney disease

Bronchiectasis type

Cylindrical

Varicose

Cystic

Lung involvement

One lung

Both lungs

Spirometry patterns

FVC, L

\section{Total}

$\mathrm{N}=506$

66 (53-79)

$254(50.2)$

$23.0(19.3-26.7)$

176 (34.8)

120 (23.7)

$15(3.0)$

261 (51.6)

82 (16.2)

191 (37.7)

64 (12.6)

$16(3.2)$

$15(3.0)$

$358(70.8)$

12 (2.4)

$315(62.3)$

$152(30.0)$

$354(70.0)$

$2.6(2.0-3.3)$

Data are presented as the median (interquartile range) or numbers (\%).

Abbreviations: $\mathrm{BMI}$, body mass index; $\mathrm{COPD}$, chronic obstructive pulmonary disease; TB, tuberculosis; FVC, forced vital capacity; $F_{E V}$, forced expiratory volume in 1 second 


\begin{tabular}{|c|c|}
\hline Variables & Total \\
\hline FVC, \% predicted & $84(70-95)$ \\
\hline $\mathrm{FEV}_{1}, \mathrm{~L}$ & $69(55-78)$ \\
\hline $\mathrm{FEV}_{1}, \%$ predicted & $1.7(1.2-2.2)$ \\
\hline $\mathrm{FEV}_{1} / \mathrm{FVC}, \%$ & $73(56-90)$ \\
\hline Microbiology & $164(32.4)$ \\
\hline Pseudomonas aeruginosa & $91(18.0)$ \\
\hline Haemophilus influenzae & $12(2.4)$ \\
\hline Staphylococcus aureus & $25(4.9)$ \\
\hline Klebsiella pneumoniae & $43(8.5)$ \\
\hline Streptococcus pneumoniae & $12(2.4)$ \\
\hline Others $^{*}$ & $52(10.3)$ \\
\hline Non-tuberculous mycobacteria & $50(10.1)$ \\
\hline MAC & $42(8.3)$ \\
\hline MABC & $8(1.6)$ \\
\hline \multicolumn{2}{|c|}{ Data are presented as the median (interquartile range) or numbers (\%). } \\
\hline \multicolumn{2}{|c|}{$\begin{array}{l}\text { Abbreviations: BMI, body mass index; COPD, chronic obstructive pulmonary disease; TB, tuberculosis; } \\
\text { FVC, forced vital capacity; FEV } 1 \text {, forced expiratory volume in } 1 \text { second }\end{array}$} \\
\hline
\end{tabular}

As shown in Table 2, 152 patients (30.0\%) with bronchiectasis had normal ventilation, 261 patients (51.6\%) had obstructive ventilatory disorder, and 93 patients (18.4\%) had restrictive ventilatory disorder. The median ages of patients with normal ventilation, obstructive ventilatory disorder, and restrictive ventilatory disorder were 66 (58-72), 68 (62-75), and 64 (56-71) years, respectively $(P<0.001)$. The proportion of males was highest in patients with obstructive ventilatory disorder, followed by those with normal ventilation and those with restrictive ventilatory disorder $(62.1 \%, 41.4 \%$, and $31.2 \%$, respectively, $P$ $<0.001)$. The proportion of current- or ex-smokers was higher among patients with obstructive ventilatory disorders compared to that in patients with normal ventilation or restrictive ventilatory disorders (43.7\%, $28.3 \%$, and $20.4 \%$, respectively, $P<0.001)$. The prevalence of COPD ( $100 \%)$, asthma $(21.8 \%)$, diabetes mellitus $(16.9 \%)$, and malignancy $(16.1 \%)$ was the highest in patients with obstructive ventilatory disorder from amongst the entire study population. Most of the microorganisms were cultured in the obstructive ventilatory disorder, followed by restrictive ventilatory disorder and normal ventilation. $(41.4 \%, 32.3 \%$ and $15.8 \%$, respectively, $P<0.001)$. The frequency of culturing for P. aeruginosa $(23.8 \%, 17.2 \%$ and $8.6 \%$, 
respectively, $P<0.001)$, E. coli (5.4\%, 3.2\%, and $0 \%$, respectively, $P<0.001)$, and others $(10.3 \%, 6.5 \%$ and $1.3 \%$, respectively, $P<0.001)$ was significantly higher in the obstructive ventilatory disorder and restrictive ventilatory disorder than that in the normal ventilation. The prevalence of NTM (20.4\%) was the highest in patients with restrictive ventilatory disorder from amongst the entire study population. 
Table 2

Baseline characteristics of patients with bronchiectasis according to spirometric pattern.

\begin{tabular}{|c|c|c|c|c|}
\hline Variables & Normal & Obstructive & Restrictive & P-value \\
\hline & $N=152$ & $N=261$ & $\mathrm{~N}=93$ & \\
\hline Age, years & $66(58-72)$ & $68(62-75)^{a}$ & $64(56-71)^{\mathrm{c}}$ & $<0.001$ \\
\hline Sex, male & $63(41.4)$ & $162(62.1)^{\mathrm{a}}$ & $29(31.2)^{c}$ & $<0.001$ \\
\hline BMI $\left(\mathrm{kg} / \mathrm{m}^{2}\right)$ & $23.4(21.1-25.5)$ & $22.9(20.4-25.2)$ & $22.5(19.9-25.6)$ & 0.275 \\
\hline \multicolumn{5}{|l|}{ Smoking history } \\
\hline Current or ex-smoker & $43(28.3)$ & $114(43.7)^{\mathrm{a}}$ & $19(20.4) c$ & $<0.001$ \\
\hline Previous history of TB & $29(19.1)$ & $68(26.1)$ & $23(24.7)$ & 0.266 \\
\hline Previous history of pertussis & $4(2.6)$ & $5(1.9)$ & $6(6.5)$ & 0.083 \\
\hline \multicolumn{5}{|l|}{ Comorbidities } \\
\hline COPD & 0 & $261(100)^{a}$ & $0^{c}$ & $<0.001$ \\
\hline Asthma & $20(13.2)$ & $57(21.8)^{\mathrm{a}}$ & $5(5.4)^{\mathrm{C}}$ & 0.001 \\
\hline Cardiovascular disease & $46(30.3)$ & $114(43.7)$ & $31(33.3)$ & 0.016 \\
\hline Diabetes mellitus & $13(8.6)$ & $44(16.9)^{\mathrm{a}}$ & $7(7.5)^{\mathrm{c}}$ & 0.013 \\
\hline Chronic liver disease & $4(2.6)$ & $8(3.1)$ & $4(4.3)$ & 0.763 \\
\hline Chronic kidney disease & $1(0.7)$ & $10(3.8)$ & $4(4.3)$ & 0.131 \\
\hline \multicolumn{5}{|l|}{ Spirometry } \\
\hline FVC, L & $2.9(2.4-3.5)$ & $2.5(1.9-3.4)^{a}$ & $2.0(1.6-2.5)^{b, c}$ & $<0.001$ \\
\hline FVC, \% predicted & $94(87-102)$ & $82(68-94)^{a}$ & $68(59-74)^{b, c}$ & $<0.001$ \\
\hline $\mathrm{FEV}_{1}, \mathrm{~L}$ & 78 (74-81) & $56(48-64)^{a}$ & $79(74-86)^{c}$ & $<0.001$ \\
\hline $\mathrm{FEV}_{1}, \%$ predicted & $2.22(1.9-2.7)$ & $1.30(1.0-1.8)^{\mathrm{a}}$ & $1.6(1.23-2.1)^{\mathrm{b}, \mathrm{c}}$ & $<0.001$ \\
\hline $\mathrm{FEV}_{1} / \mathrm{FVC}, \%$ & $98(88-108)$ & $61(45-74)^{a}$ & $69(61-79)^{b, c}$ & $<0.001$ \\
\hline NTM & $15(9.9)$ & $17(6.5)$ & $19(20.4)^{b, c}$ & 0.001 \\
\hline MAC & 13/15 (86.7) & 15/17 (88.2) & 14/19 (73.7) & 0.454 \\
\hline MABC & 2/15 (13.3) & $2 / 17(11.8)$ & 4/19 (21.1) & 0.167 \\
\hline Microbiology & $24(15.8)$ & $108(41.4)^{\mathrm{a}}$ & $30(32.3)^{b}$ & $<0.001$ \\
\hline
\end{tabular}




\begin{tabular}{|c|c|c|c|c|}
\hline Variables & Normal & Obstructive & Restrictive & P-value \\
\hline P. aeruginosa & $13(8.6)$ & $62(23.8)^{a}$ & $16(17.2)^{b}$ & 0.001 \\
\hline H. influenzae & $1(0.7)$ & $9(3.4)$ & $2(2.2)$ & 0.196 \\
\hline S. aureus & $3(2.0)$ & $18(6.9)^{\mathrm{a}}$ & $4(4.3)$ & 0.080 \\
\hline K. pneumoniae & $9(5.9)$ & $26(10.0)$ & $8(8.6)$ & 0.364 \\
\hline S. pneumoniae & $1(0.7)$ & $9(3.4)$ & $2(2.2)$ & 0.196 \\
\hline Others & $2(1.3)$ & $27(10.3)^{a}$ & $6(6.5)^{\mathrm{b}}$ & 0.002 \\
\hline \multicolumn{5}{|c|}{ Data are presented as the median (interquartile range) or numbers (\%). } \\
\hline \multicolumn{5}{|c|}{ One patient had co-infection with MAC and M. abscessus. } \\
\hline \multicolumn{5}{|c|}{$\begin{array}{l}\text { Others had multidrug-resistant bacteria identified in hospital-acquired pneumonia (e.g., Acinetobacter } \\
\text { baumannii, Stenotrophomonas maltophilia). }\end{array}$} \\
\hline \multicolumn{5}{|c|}{ Due to Bonferroni correction with three comparisons, the p-value of 0.05 corresponds to $0.17(0.05 / 3)$} \\
\hline \multicolumn{5}{|c|}{ aThere was a significant difference between normal lung function and the obstructive pattern. } \\
\hline \multicolumn{5}{|c|}{${ }^{\mathrm{b}}$ There was a significant difference between normal lung function and the restrictive pattern. } \\
\hline \multicolumn{5}{|c|}{${ }^{\mathrm{C}}$ There was a significant difference between the obstructive and restrictive patterns. } \\
\hline \multicolumn{5}{|c|}{ 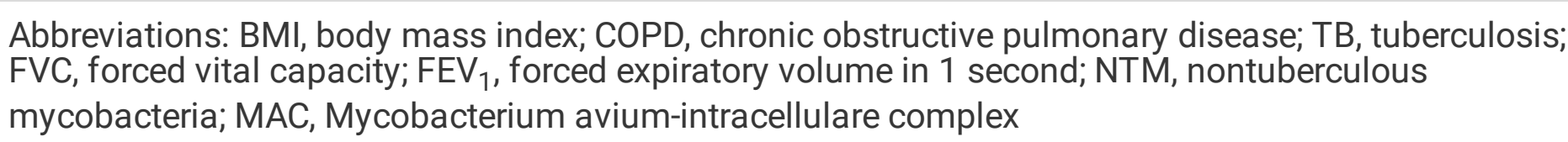 } \\
\hline
\end{tabular}

Respiratory symptoms, radiological features, and disease severity scales in patients with bronchiectasis according to the spirometric pattern

As shown in Table 3, the modified Medical Research Council (mMRC) was the highest in patients with obstructive ventilatory disorder, followed by those with restrictive ventilatory disorder and normal ventilation [1 (1-2), 1 (0-2), and 0 (0-1), respectively; $P<0.001$ ]. The modified Reiff score [6 (3-10), $P<0.001)$ ]; FACED score [3 (1-4), $P<0.001$ ], and BSI score [8 (5-11), $P<0.001$ ] were significantly higher in the obstructive ventilatory disorder group than those in the restrictive ventilatory disorder and normal ventilation groups. There was no significant correlation between the lung function pattern and bronchiectasis type. The proportion of bilateral lung involvement was the highest in patients with obstructive ventilatory disorder, followed by those with restrictive ventilatory disorder and normal ventilation $(79.7 \%, 72.0 \%$, and $52.0 \%$, respectively, $P<0.001)$. The disease severity indices, i.e., the $\mathrm{BSI}$ and FACED scores, were the highest in patients with obstructive ventilatory disorder, followed by those with restrictive ventilatory disorder and normal ventilation [8 (5-11), 6 (4-9) and $4(2-7)$, respectively, $P<0.001$ for the BSI score; 3 (1-4), 1 (1-3) and 1 (0-2), respectively, $P<0.001$ for the FACED score]. 
Table 3

Respiratory symptoms, radiological features and disease severity scales of patients with bronchiectasis according to spirometric patterns.

\begin{tabular}{|c|c|c|c|c|c|}
\hline Variables & $\begin{array}{l}\text { Total } \\
\mathrm{N}=506\end{array}$ & $\begin{array}{l}\text { Normal } \\
\mathrm{N}=152\end{array}$ & $\begin{array}{l}\text { Obstructive } \\
\mathrm{N}=261\end{array}$ & $\begin{array}{l}\text { Restrictive } \\
\mathrm{N}=93\end{array}$ & P-value \\
\hline $\mathrm{mMRC}$ & $0(1-2)$ & $0(0-1)$ & $1(1-2)^{a, c}$ & $1(0-2)^{b, c}$ & $<0.001$ \\
\hline Hemoptysis & $150(29.6)$ & $45(29.6)$ & $79(30.3)$ & $26(28.0)$ & 0.916 \\
\hline BAE & $36 / 150$ (24.0) & $8 / 45(17.8)$ & $20 / 79(25.3)$ & $8 / 26(30.8)$ & 0.431 \\
\hline Modified Reiff score & $5(3-8)$ & $3(2-6)$ & $6(3-10)^{a}$ & $5(3-8)^{b}$ & $<0.001$ \\
\hline \multicolumn{6}{|l|}{ Bronchiectasis type } \\
\hline Cylindrical & $358(70.8)$ & $106(69.7)$ & $185(70.9)$ & $67(72.0)$ & 0.926 \\
\hline Varicose & $12(2.4)$ & $3(2.0)$ & $7(2.7)$ & $2(2.2)$ & 0.890 \\
\hline Cystic & $315(62.3)$ & $87(57.2)$ & $167(64.0)$ & $61(65.6)$ & 0.301 \\
\hline \multicolumn{6}{|l|}{ Lung involvement } \\
\hline One lung & $152(30.0)$ & $73(48.0)$ & $53(20.3)^{a}$ & $26(28.0)^{b}$ & $<0.001$ \\
\hline Both lungs & $354(70.0)$ & $79(52.0)$ & $208(79.7)^{a}$ & $67(72.0)^{\mathrm{b}}$ & $<0.001$ \\
\hline FACED score & $2(1-3)$ & $1(0-2)$ & $3(1-4)^{a, c}$ & $1(1-3)^{b, c}$ & $<0.001$ \\
\hline FACED score risk class & & & & & $<0.001$ \\
\hline Mild (0-2) & 303 (59.9) & $122(80.3)$ & $114(43.7)^{a, c}$ & $67(72.0)^{\mathrm{b}, \mathrm{c}}$ & \\
\hline Moderate (3-4) & $158(31.2)$ & 30 (197) & $106(40.6)^{a, c}$ & $22(23.7)^{b, c}$ & \\
\hline Severe (5-7) & $45(8.9)$ & 0 & $41(15.7)^{a, c}$ & $4(4.3)^{\mathrm{b}, \mathrm{c}}$ & \\
\hline BSI score & $6(4-10)$ & $4(2-7)$ & $8(5-11)^{a, c}$ & $6(4-9)^{b, c}$ & $<0.001$ \\
\hline BSI score risk class & & & & & $<0.001$ \\
\hline Mild (0-4) & $179(35.4)$ & $80(52.6)$ & $63(24.1)^{a, c}$ & $36(38.7)^{b, c}$ & \\
\hline Moderate (5-8) & $211(41.7)$ & $62(40.8)$ & $109(41.8)^{\mathrm{a}, \mathrm{c}}$ & $40(43.0)^{b, c}$ & \\
\hline Severe $(9+)$ & $116(22.9)$ & $10(6.6)$ & $89(34.1)^{a, c}$ & $17(18.3)^{b, c}$ & \\
\hline Follow-up duration, months & $28(6-55)$ & $17(2-42)$ & $36(12-69)^{a, c}$ & $17(4-43)^{c}$ & $<0.001$ \\
\hline
\end{tabular}




\begin{tabular}{|llllll|}
\hline Variables & Total & Normal & Obstructive & Restrictive & P-value \\
& $\mathrm{N}=506$ & $\mathrm{~N}=152$ & $\mathrm{~N}=261$ & $\mathrm{~N}=93$ & \\
\hline
\end{tabular}

One person had a co-infection of MAC and M. abscessus.

Others were multidrug-resistant bacteria identified in Hospital-acquired pneumonia (e.g., Acinetobacter baumannii, Stenotrophomonas maltophilia).

Due to Bonferroni correction with three comparisons, the $p$-value of 0.05 corresponds to $0.17(0.05 / 3)$.

${ }^{a}$ There was a significant difference between normal lung function and the obstructive pattern.

${ }^{b}$ There was a significant difference between normal lung function and the restrictive pattern.

${ }^{\mathrm{C}}$ There was a significant difference between the obstructive and restrictive patterns.

Abbreviations: mMRC, Modified Medical Research Council; BAE, bronchial artery embolization; BSI, Bronchiectasis Severity Index; NTM, nontuberculous mycobacteria; MAC, Mycobacterium aviumintracellulare complex

\section{Correlation between the modified Reiff score and lung function}

As shown in Figure 2, the $\mathrm{FVC} \%, \mathrm{FEV}_{1} / \mathrm{FVC} \%$, and $\mathrm{FEV}_{1} \%$ declined significantly with the elevation in the modified Reiff score $\left(P<0.001\right.$ for all). The $\mathrm{FEV}_{1}$ declined significantly with the increase in the BSI score $(\mathrm{r}=-0.442$, respectively; $P<0.001)$ in the obstructive pulmonary disorder group. The FVC decreased significantly with the increase in the BSI score $(r=-0.363, P<0.001)$ in the restrictive pulmonary disorder group (Supplemental figure 1).

\section{Discussion}

To the best of our knowledge, this is the first study to evaluate the clinical characteristics, radiological features, and disease severity according to the spirometric pattern in patients with bronchiectasis. Approximately $52 \%$ and $18 \%$ patients with bronchiectasis had obstructive and restrictive ventilatory disorders, respectively. The frequency of dyspnea, higher disease severity (including high BSI and FACED scores), higher sputum culture positivity for Pseudomonas aeruginosa, and greater radiological severity (i.e., high modified Reiff scores) were predominant in bronchiectasis accompanied by obstructive ventilatory disorder. On the other hand, bronchiectasis with restrictive ventilatory disorder was associated with a high prevalence of NTM disease. There was no significant correlation between the spirometric patterns and the type of bronchiectasis; however, lung function decreased with the increase in the modified Reiff score.

Patients with bronchiectasis frequently experience structural and functional lung damage that vary in severity $2,8,9$. Our notable finding was that more than half of patients $(52 \%)$ with bronchiectasis showed obstructive ventilatory disorder. In previous studies, approximately up to $35 \%$ of bronchiectasis were 
known to experience obstructive ventilatory disorders ${ }^{5,12,20-22}$. Bronchiectasis is known to manifest as an obstructive ventilatory disorder due to continuous airway destruction and distortion caused by chronic inflammation and abnormal mucociliary clearance ${ }^{2}$. In our study, the bilateral lung involvement was high at $70 \%$ in patients with bronchiectasis. Bilateral lung involvement resulted in widespread inflammation of the bronchial tree and damage to the lung parenchyma, which is believed to have resulted in pulmonary impairment of obstructive ventilatory disorder. Additionally, patients with bronchiectasis may also experience restrictive ventilatory disorder. Bronchiectasis accompanied by atelectasis and pleural disease, parenchymal scarring, and peribronchial fibrosis is thought to be associated with restrictive ventilatory disorder ${ }^{11}$. In contrast with obstructive ventilatory disorder, only a few studies have described restrictive ventilatory disorder and the presumable prevalence was reported to be $8-15 \%$ among patients with bronchiectasis $^{12,20,22}$. Our study also reported a similar result, i.e., $18 \%$ of patients with bronchiectasis had restrictive ventilatory disorder; however, verification through a large-scale study is necessary since it accounted for a small proportion of the study population.

Our notable finding was that bronchiectasis with obstructive ventilatory disorder was characterized by greater symptoms of dyspnea, disease severity and radiological severity. We measured the degree of dyspnea using the mMRC score, which was significantly higher in bronchiectasis with obstructive ventilatory disorder. $\mathrm{mMRC}$ is a subjective measure of dyspnea in COPD, which is known to be associated with the decrease in $\mathrm{FEV}_{1}{ }^{23}$. Similarly, in our study, the degree of dyspnea as measured by the mMRC score was higher in the obstructive ventilatory disorder group with low $\mathrm{FEV}_{1}$. Moreover, we evaluated disease severity using the BSI and FACED scores. The FACED score $\left[\mathrm{FEV}_{1} \%\right.$ predicted $(\mathrm{F})$, age $(A)$, chronic colonization by Pseudomonas aeruginosa (C), extension of the disease by radiological assessment $(E)$ and dyspnea (D) $]^{9}$ is a five-point instrument that predicts the probability of all-cause mortality after 5 years of follow-up, whereas the $\mathrm{BSI}^{8}$ is a seven-point scale that identifies patients with bronchiectasis at risk for future mortality, hospitalization, exacerbations, and deterioration in the quality of life. Our study revealed high disease severity and poor prognosis, as measured using the BSI and FACED, in bronchiectasis with obstructive ventilatory disorder. These results may be related to low BMI, low FEV ${ }_{1}$, high mMRC score, and high Pseudomonas aeruginosa infection, which are the clinical features of bronchiectasis with obstructive ventilatory disorder ascertained by our study. Although most studies have reported that the $\mathrm{FEV}_{1}$ decreases with the increase in CT severity ${ }^{11,24,25}$, no study has analyzed the relationship between $\mathrm{CT}$ severity and lung function in bronchiectasis with obstructive ventilatory disorder. Our study is the first to demonstrate the impairment in lung function, including FVC and FEV 1 , and CT severity using the modified Reiff score in bronchiectasis with obstructive ventilatory disorder. Therefore, these findings emphasize the importance of the management of lung function in bronchiectasis with obstructive ventilatory disorder.

Another interesting finding of our study was that there was no significant correlation between the spirometric pattern and radiological type of bronchiectasis. However, the increase in the radiological severity, which was measured by the modified Reiff score, was accompanied by the increase in the 
severity of impairment of lung function. Bronchiectasis is known to manifest as a combination of three morphologies, viz., cylindrical, varicose, and cystic ${ }^{26}$. Few studies have investigated the relationship between the type of bronchiectasis and the spirometric pattern. In contrast with our study, other studies have suggested that cylindrical bronchiectasis is associated with obstructive ventilatory disorder ${ }^{22,27}$. However, it is difficult to generalize the results of studies that incorporated a small patient population. Thus, large-scale research on the association between the radiological features of bronchiectasis and lung function is warranted. Our study also showed that the severity of lung function impairment increased with the radiological extent of bronchiectasis. Inflammation and destruction of the lung parenchyma and airways become more severe with the expansion in the extent of bronchiectasis, which may lead to a decrease in lung function. Other studies have also reported similar results ${ }^{11,24}$. Thus, we can infer that the extent of bronchiectasis, rather than the type of bronchiectasis, affects lung function.

This study had several limitations. First, it was conducted at a single center in South Korea. Second, its retrospective and observational design could have introduced potential biases. For example, the proportion of patients with obstructive ventilatory disorder was high in this bronchiectasis population, possibly because this study was conducted in patients with bronchiectasis who underwent pulmonary function testing. Third, the BSI and FACED scores were high in the bronchiectasis with obstructive ventilatory disorder group. $\mathrm{FEV}_{1}$ is included in the BSI and FACED evaluation criteria, which may be a confounding factor. However, our study showed that low BMI, high mMRC score, and high prevalence of Pseudomonas aeruginosa infection were clinical features associated with the severity of bronchiectasis with obstructive ventilatory disorder, even after excluding these confounding factors.

In conclusion, among patients with bronchiectasis, $52 \%$ had obstructive ventilatory disorder and $18 \%$ had restrictive ventilatory disorder. Bronchiectasis with obstructive ventilatory disorder had more dyspnea, and greater disease and radiological severity. The extent of bronchiectasis rather than radiologic type of bronchiectasis affects lung function impairment, however more research is needed.

\section{Methods}

\section{Study setting and patients population}

We retrospectively reviewed the medical records of 1369 patients at Chungbuk National University Hospital (a 793-bed referral hospital) in Cheongju, Republic of Korea, in whom bronchiectasis was diagnosed between January 2014 to August 2021. Of those patients, those not undergoing pulmonary function test $(n=863)$ were excluded, a total of 506 patients were included in the study. We classified patients with bronchiectasis into three groups according to spirometric patterns (Figure 1).

\section{Ethics approval and consent to participate}

The study was approved by the Ethical Committee of the "Chungbuk National University Hospital" in compliance with the ethical principles (IRB No. CBNUH 2021-09-023). This study was conducted in accordance with the amended Declaration of Helsinki (as revised in 2013). Patient information was 
anonymized and de-identified prior to analysis. Therefore, the need for informed consent was waived owing to the retrospective nature of the study.

\section{Pulmonary function tests}

Pre-bronchodilator and post-bronchodilator spirometry was performed according to American Thoracic Society/European criteria ${ }^{13}$. The absolute values of $\mathrm{FEV}_{1}$ and forced vital capacity (FVC) were recorded, and the percentage of predicted values for $\mathrm{FEV}_{1}$ and $\mathrm{FVC}$ were calculated using an automatic calculator with a reference equation obtained from a representative Korean sample ${ }^{14}$. Normal ventilation was defined as pre-bronchodilator $\mathrm{FEV}_{1} / \mathrm{FVC} \geq 0.70$ and $\mathrm{FVC} \geq 80 \%$ predicted. Obstructive ventilatory disorder was defined as pre-bronchodilator $\mathrm{FEV}_{1} / \mathrm{FVC}<0.70$. Restrictive ventilatory disorder was defined as $\mathrm{FEV}_{1} / \mathrm{FVC} \geq 0.7$ and $\mathrm{FVC}<80 \%$ predicted $^{15}$.

\section{Bronchiectasis severity: Radiological and disease severity}

The radiological severity of bronchiectasis was measured using the modified Reiff score ${ }^{16}$. Chest computed tomography (CT) was used to evaluate the radiological extent of bronchiectasis in all participants. The number of lobes involved (the lingula was considered to be a separate lobe) and degree of dilatation (tubular: 1 , varicose: 2 , and cystic: 3 ) were determined by three researchers, including two pulmonologists (SHK and BY) and one radiologist (JYY), based on the consensual interpretation of the chest CT image. The FACED and BSI scores were calculated to assess the clinical status and severity of the bronchiectasis, based on previous studies ${ }^{8,9}$.

\section{Microbiology}

Spontaneous sputum or lower tract specimens (bronchoalveolar lavage) were obtained from all patients. The specimens were subjected to microbiological analyses according to standard methods ${ }^{17}$. Conventional semi-qualitative bacterial and fungal cultures were performed. All samples underwent initial Gram staining prior to sputum culture, if the Murray and Washington criteria were met ${ }^{18}$. Nontuberculous mycobacteria (NTM) lung disease was diagnosed using the microbiological criteria provided by the American Thoracic Society and Infectious Disease Society of America; (1) two positive sputum cultures, (2) one positive bronchial wash or lavage, (3) compatible mycobacterial histological features such as granulomatous inflammation, and positive results on acid-fast bacilli lung biopsy and/or lung biopsy culture, and (4) more than one positive sputum culture or bronchial wash ${ }^{19}$.

\section{Statistical analysis}

Data were presented as the median and interquartile range (IQR) for continuous variables and frequency (percentage) for categorical variables. Continuous variables were compared using the Kruskal-Wallis test and the Pearson chi-squared test or Fisher exact test was used for categorical variables. Moreover, $\mathrm{p}$ values for comparing the three ventilatory disorder groups were adjusted using Bonferroni correction. 
Spearman's rho correlation method was used to confirm the existence of a correlation between the BSI score and lung function tests. All tests were two-sided, and p-values $<0.05$ were considered statistically significant. All statistical analyses were conducted using IBM SPSS Statistics for Windows (version 21.0; IBM Corp., Armonk, NY, USA) and STATA (version 15; Stata Corp., College Station, TX, USA).

\section{Declarations}

\section{Data Availability}

The data that support the findings of this study are available from the corresponding author upon reasonable request.

\section{Acknowledgements}

This work was supported by grants from the National Research Foundation of Korea (2020R1A5A2017476) and Chungbuk national university hospital in 2021.

\section{Author Contributions}

Conceptualization: Choe K.H., Kim E.G., Yang B. Investigation: Kim S.H., Yang B. Formal analysis: Kim S.H., Yang B., Yoo J.Y. Methodology: Choe K.H., Kim S.H., Yang B. Supervision: Kim E.G., Yoo J.Y. Writingoriginal draft: Choe K.H., Kim S.H., Yang B. Writing-review \& editing: Choe K.H., Kim S.H., Yang B.H., Yoo J.Y., Cho J.Y., Kang H., Shin Y.M., Lee K.M.

\section{Competing interests}

The authors declare no competing interests.

\section{References}

1. Cole, P. Inflammation: a two-edged sword--the model of bronchiectasis. Eur. J. Respir. Dis. Suppl. 1986; 147: 6-15.

2. Chalmers, J.D., Chang, A.B., Chotirmall, S.H., Dhar, R. \& McShane, P.J. Bronchiectasis. Nat. Rev. Dis. Primer. 2018; 4: 1-18.

3. Ooi, G.C. et al. High-resolution CT quantification of bronchiectasis: clinical and functional correlation. Radiology. 2002; 225: 663-72.

4. Sheehan, R.E. et al. A comparison of serial computed tomography and functional change in bronchiectasis. Eur. Respir. J. 2002; 20: 581-7.

5. Lee, J-H., Kim, Y-K., Kwag, H-J. \& Chang, J-H. Relationships between high-resolution computed tomography, lung function and bacteriology in stable bronchiectasis. J. Korean. Med. Sci. 2004; 19: 62-8.

6. Whitwell, F. A study of the pathology and pathogenesis of bronchiectasis. Thorax. 1952; 7: 213. 
7. Culiner, M.M. Obliterative bronchitis and bronchiolitis with bronchiectasis. Dis. Chest. 1963; 44: 35161.

8. Chalmers, J.D. et al. The bronchiectasis severity index. An international derivation and validation study. Am. J. Respir. Crit. Care. Med. 2014; 189: 576-85.

9. Martínez-García, M.Á. et al. Multidimensional approach to non-cystic fibrosis bronchiectasis: the FACED score. Eur. Respir. J. 2014; 43: 1357-67.

10. Guan, W.J., et al. Characterization of lung function impairment in adults with bronchiectasis. PLoS. One. 2014;9: e113373.

11. Habesoglu, M.A., Tercan, F., Ozkan, U. \& Fusun, E.O. Effect of radiological extent and severity of bronchiectasis on pulmonary function. Multidiscip. Respir. Med. 2011; 6: 1-7.

12. Radovanovic, D. et al. A comprehensive approach to lung function in bronchiectasis. Respir. Med. 2018; 145: 120-9.

13. Miller, M.R. et al. Standardisation of spirometry. Eur. Respir. J. 2005; 26: 319-38.

14. Choi, J.K., Paek, D. \& Lee, J.O. Normal predictive values of spirometry in Korean population. Tuberc Respir Dis 2005; 58: 230-42.

15. Sim. Y.S. et al. Spirometry and Bronchodilator Test. Tuberc Respir Dis 2017; 80: 105-12.

16. Reiff, D.B., Wells, A.U., Carr, D.H., Cole, P.J. \& Hansell, D.M. CT findings in bronchiectasis: limited value in distinguishing between idiopathic and specific types. AJR. Am. J. Roentgenol.1995; 165: 261-7.

17. Patel, J.B., Cockerill, F. \& Bradford, P.A. Performance standards for antimicrobial susceptibility testing; Twenty-Fifth Informational Supplement. 2015.

18. Murray, P.R. \& Washington, J.A. Microscopic and bacteriologic analysis of expectorated sputum. Mayo Clin. Proc. 1975; 50: 339-44.

19. Griffith, D.E. et al. An official ATS/IDSA statement: diagnosis, treatment, and prevention of nontuberculous mycobacterial diseases. Am. J. Respir. Crit. Care. Med. 2007; 175: 367-416.

20. Yang, B. et al. Factors associated with bronchiectasis in Korea: a national database study. Ann. Transl. Med. 2020; 8:1350.

21. Lee, H. et al. Characteristics of bronchiectasis in Korea: First data from the Korean Multicentre Bronchiectasis Audit and Research Collaboration registry and comparison with other international registries. Respirology. 2021, 26: 619-21.

22. Lynch, D.A. et al. Correlation of CT findings with clinical evaluations in 261 patients with symptomatic bronchiectasis. AJR. Am. J. Roentgenol. 1999; 173: 53-8.

23. Bestall, J.C. et al. Usefulness of the Medical Research Council (MRC) dyspnoea scale as a measure of disability in patients with chronic obstructive pulmonary disease. Thorax. 1999, 54: 581-6.

24. Roberts, H.R. et al. Airflow obstruction in bronchiectasis: correlation between computed tomography features and pulmonary function tests. Thorax. 2000; 55: 198-204.

25. Ibrahim, R.M., Elnekeidy, A., Rizk, A., Yossef, A. \& Abdelrahman, S. Correlation between a proposed MDCT severity score of bronchiectasis and pulmonary function tests. Egypt. J. Radiol. Nucl. Med. 
2016; 47: 413-20.

26. Milliron, B., Henry, T.S., Veeraraghavan, S., \& Little, B.P. Bronchiectasis: mechanisms and imaging clues of associated common and uncommon diseases. Radiographics. 2015; 35: 1011-30.

27. Nicotra, M.B., Rivera, M., Dale, A.M., Shepherd, R. \& Carter, R. Clinical, pathophysiologic, and microbiologic characterization of bronchiectasis in an aging cohort. Chest. 1995, 108: 955-61.

\section{Figures}

\section{Figure 1}

Flow chart of study population

Figure 2

Correlation between the modified Reiff score and lung function

(a) Obstructive pattern

(b) Restrictive pattern

\section{Supplementary Files}

This is a list of supplementary files associated with this preprint. Click to download.

- supplementalfigure.jpg 